\title{
Cambio de gestión administrativa en los gobiernos sub nacionales y su incidencia en los sistemas administrativos: Región Amazonas, 2018-2019
}

Change of administrative management in sub-national governments and its impact on administrative systems: Amazonas region, 2018-2019

\author{
Línea de investigación: Sistemas administrativos del Estado
}

\section{AUTOR: \\ Ms. Ing. Heisely Mori \\ Pelaez \\ Maestro en Gestión para el Desarrollo \\ Sustentable, Universidad Nacional Toribio Rodríguez de Mendoza de Amazonas, Chachapoyas - Perú \\ Docente de la \\ Universidad Nacional \\ Toribio Rodríguez de \\ Mendoza de Amazonas \\ ORCID: \\ https://orcid.org/0000- 0003-1722-998X \\ Correo: \\ hmpamazonas@gmail.c om}

\section{RESUMEN:}

La presente investigación evalúa las posibles consecuencias de reprogramar de la fecha de cambio de gestión administrativa de los gobiernos locales y regionales peruanos, del 31 de diciembre al 28 de julio evaluado en los impactos sobre los sistemas administrativos del Estado. Para ello se realizó un panel de expertos o método Delphi, con veinte funcionarios de la región Amazonas provenientes del gobierno regional y cinco municipalidades, que participaron en al menos un cambio de gestión administrativa entre los años 2018 y 2019. Se sistematizaron los resultados del método Delphi identificando las debilidades y fortalezas, así como las ventajas y desventajas de la propuesta sobre los sistemas administrativos del Estado peruano. Se observó que la propuesta impactaba notablemente en los sistemas administrativos de: Gestión de Recursos Humanos, Abastecimiento, Presupuesto Público, Tesorería, Contabilidad, Inversión Pública y Planeamiento Estratégico. Luego se aplicó el método AHP (Proceso de Análisis Jerárquico, del inglés Analytical Hierarchy Process) para determinar la prioridad de las propuestas, empleando nueve criterios de evaluación distribuidos en cuatro factores: Sociales, económicos, institucionales y legales. La aplicación del método AHP define que los beneficios para los ciudadanos peruanos son mayores cuando se realiza el cambio de gestión administrativa el 28 de julio.

Palabras clave: Administración pública, Administración local, Organización, gestión

\section{ABSTRACT:}

The present research evaluates the possible consequences of rescheduling the date of change of administrative management of the Peruvian local and regional governments, from December 31 to July 28 , evaluated in the impacts on the administrative systems of the State. For this, a panel of experts or Delphi method was carried out, with twenty officers from the Amazonas region coming from the regional government and five municipalities, who participated in at least one change of administrative management between 2018 and 2019. The results of the Delphi method were systematized, identifying the weakness and strengths, as well as the advantages and disadvantages of the proposal on the administrative systems of the Peruvian State. It was observed that the proposal had a significant impact on the administrative systems of: Human Resources Management, Supplying, Public Budget, Treasury, Accounting, Public Investment and Strategic Planning. Then the AHP (Analytical Hierarchy Process) methodology was applied to determine the priority of the proposals, using nine assessment criteria distributed in four factors: Social, economic, institutional and legal. The application of the AHP method defines that the benefits for Peruvian citizens are greater when the change of administrative management is made on July 28.

Keywords: Public administration, Local government, Organization and management 


\section{INTRODUCCIÓN}

Según la Constitución Política del Perú (1993), el país se encuentra integrado por regiones, departamentos, provincias y distritos, organizado en tres niveles de gobierno que son nacional, regional y local. Para fines administrativos, políticos y económicos los gobiernos regionales y locales son autónomos buscando fomentar el desarrollo en los ámbitos de su competencia (Ley №27867, Orgánica de Gobiernos Regionales y Ley № 27972, Orgánica de Municipalidades).

La normativa electoral establece que las elecciones para gobiernos regionales y locales se realizan cada cuatro años, donde se eligen gobernador y vice gobernador, consejeros regionales, alcaldes provinciales y distritales y regidores municipales (Ley № 27683, de Elecciones Regionales y Ley N²6864, de Elecciones Municipales).

Claros (2014) analiza el proceso electoral del año 2014, donde se eligieron autoridades de gobiernos regionales y locales, encontrándose políticos implicados en diversos casos de corrupción y malas prácticas, hechos que podrían peligrar la institucionalidad de estos gobiernos sub nacionales por las conductas observadas de las nuevas autoridades. El autor también rememora los procesos de transferencia de los gobiernos regionales y locales, que, si bien estos últimos se restituyeron en el Perú en el año 1980, es recién hasta el año 1998 que se norman, a través de la emisión de la Ley $N^{\circ} 26997$, Ley que establece la conformación de comisiones de transferencia de la administración municipal. Antes de esta norma, las transferencias eran realizadas sin formalidad, ocultando información, destruyendo o desapareciendo expedientes y sin plazos determinados.

Para el cambio de gestión, para el periodo 2019-2022, la Contraloría General de la República acompañó al proceso de transferencia, emitiendo la Directiva № 008-2018CG/GTN “Transferencia de la Gestión Administrativa de los Gobiernos Regionales y Gobiernos Locales", aprobada mediante Resolución de Contraloría N³48-2018-CG, publicada en junio del 2018. Esto ayuda mucho para evitar problemas en la transferencia 
de gestión, pero todavía se evidencia algunos vacíos legales, los cuales se espera sean normados en los próximos meses.

Una norma que será de particular importancia para analizar el cambio de gestión administrativa es el Decreto Legislativo N 1440, Del Sistema Nacional de Presupuesto Público, donde se norma la ejecución presupuestaria en el Perú, estableciéndolo análogamente con el año calendario, es decir inicia el 01 de enero y culmina el 31 de diciembre. Durante este periodo se captan los ingresos y se ejecutan los gastos de todas las instituciones públicas.

En las dos normas citadas se evidenciaría que noviembre y diciembre de cada año es un periodo para la transferencia de gestión, sin embargo, el 31 de diciembre es el límite para reconocer las deudas asumidas por la institución, a través del devengado correspondiente, lo cual generaría dificultades para un correcto cierre de año.

En la investigación se busca determinar una fecha óptima para la transferencia de gestión, que no perjudique la ejecución presupuestaria y favorezca la continuidad de la prestación de servicios que provee el gobierno regional y/o local y repercuta tangiblemente en beneficio de la población. Para determinar cuál es la mejor fecha para el cambio de gestión administrativa se realizó un Delphi con veinte funcionarios de gobiernos regionales y locales de la región Amazonas con quienes se analizó el funcionamiento y riesgos de los once sistemas administrativos con los que cuenta el Estado peruano, datos que luego fueron procesados siguiendo la metodología del Proceso de Análisis Jerárquico (AHP), determinando que la mejor fecha para el cambio de gestión administrativa de los gobierno sub nacionales sería el 28 de julio.

\section{MARCO TEÓRICO}

Para Asela (2015), la descentralización es un modelo de administración del Estado, que en los últimos años ha propuesto una nueva estrategia de desarrollo, partiendo desde lo pequeño hasta la nación en su conjunto. Acciones propiciadas a través de las 
dimensiones política y económica. Para la dimensión política, la descentralización centra su atención en los gobiernos sub nacionales, entendidos como divisiones territoriales administrativas a niveles regionales o locales, brindándoles la oportunidad de organizarse para proveer bienes y servicios públicos en su jurisdicción, en el marco de una autonomía fiscal. Para la dimensión económica, la descentralización busca minimizar los riesgos de la actuación del gobierno central, relacionado con el tamaño de aparato estatal, así como la ineficiencia que genera desequilibrios fiscales y sociales.

Se podría decir que el proceso de descentralización política en el Perú se inicia el año 2001, con la promulgación de la Ley N²6922, Ley Marco de Descentralización. Continuándose con el marco normativa con la promulgación de la Ley № 27783, Ley de Bases de la Descentralización en el Perú, el año 2002.

Arroyo (2017) analiza el proceso de descentralización en el Perú, a quince años de iniciado, y recapitula que, sobre los intentos normativos y administrativos realizados, el Estado multinivel todavía no funciona como se había planificado, si bien es cierto, se ha avanzado en descentralizar funciones, las instituciones descentralizadas no se desempeñan eficientemente, evidenciándose a través de bajas ejecuciones presupuestales y altos índices de corrupción en los gobiernos sub nacionales.

Según estadísticas presentadas por Arroyo (2017), en el primer semestre del año 2016, la región de Tacna gastó solo el 15\% del presupuesto institucional, mientras que las regiones Pasco, Ancash y Puno, apenas superaron el 17\% de ejecución para el mismo periodo. De otro lado, en relación con la corrupción, menciona a dos ex presidentes regionales presos por corrupción y otros 18 con procesos de investigación judicial.

Por su parte, la Organisation for Economic Co-operation and Development (2017) afirma que el proceso de descentralización iniciado en el Perú el año 2002, ha logrado avances importantes en materia de descentralización política, con la elección de los gobiernos regionales y la transferencia de importantes responsabilidades al nivel sub 
nacional. Sin embargo, esto no ha ido acompañado de cambios en materia tributaria y de transferencias de recursos, tampoco de una estrategia coherente para aumentar las habilidades y capacidades a nivel sub nacional. Esta situación, sumada a la superposición de responsabilidades y competencias entre los tres niveles de gobierno y las limitadas coordinaciones horizontales y verticales, ha impedido que el país logre los objetivos de descentralización.

Para el portal Definición.de (2021), definir administración nos relaciona con el funcionamiento, la estructura y la producción de las organizaciones. La administración sería entonces la disciplina encargada de gestionar los recursos de una organización, tanto materiales, financieros y humanos, basada en criterios científicos y persiguiendo cumplir metas y objetivos concretos. Si nos referimos a la administración pública, estaremos hablando del conjunto de organismos encargados de gestionar los recursos del Estado mediante la aplicación de normas y directivas para brindar bienes y servicios a los usuarios de su jurisdicción.

Para De la Paz (2017), el concepto de administración pública está definida por atributos meramente estatales, cuyo fin fundamental es la sociedad, posibilitando su perpetuación y desarrollo. En consecuencia, hablar de administración pública es hablar de aquellas actividades que el Estado despliega con la finalidad de producir las condiciones necesarias para posibilitar la perpetuación de la sociedad, así como fomentar las condiciones necesarias para que sus elementos constitutivos se desarrollen.

R\&C Consulting (2021) define a los sistemas administrativos del estado peruano a partir de la Ley $N^{\circ} 29158$, Ley Orgánica del Poder Ejecutivo, estableciendo que son los conjuntos de principios, normas, procedimientos, técnicas e instrumentos que sirven de base para que las actividades de la administración pública se organicen. Para su realización se requiere que varias entidades los ejecuten, siguiendo los lineamientos emitidos por el Ente rector, definido al momento de establecerse el sistema. La principal 
finalidad de los sistemas administrativos es regular el uso de los recursos públicos, promoviendo las buenas prácticas de gestión administrativa.

Los sistemas administrativos del Estado peruano (Martínez, 2015) se definen a continuación, en la Tabla 1.

\section{Tabla 1}

Sistemas administrativos del estado peruano

\begin{tabular}{|c|c|c|}
\hline Sistema & Ente Rector & Descripción \\
\hline $\begin{array}{l}\text { Gestión de } \\
\text { Recursos } \\
\text { humanos }\end{array}$ & $\begin{array}{l}\text { Presidencia del Consejo } \\
\text { de Ministros y Autoridad } \\
\text { Nacional del Servicio } \\
\text { Civil }\end{array}$ & $\begin{array}{l}\text { Es el encargado de establecer, } \\
\text { desarrollar y ejecutar las políticas } \\
\text { de recursos humanos del } \\
\text { personal que brinda servicios al } \\
\text { Estado, principalmente en el } \\
\text { marco de la Ley del Servicio Civil }\end{array}$ \\
\hline Abastecimiento & $\begin{array}{l}\text { Ministerio de Economía } \\
\text { y Finanzas y Organismo } \\
\text { Supervisor de las } \\
\text { Contrataciones del } \\
\text { Estado }\end{array}$ & $\begin{array}{l}\text { Es el encargado de normar la } \\
\text { dotación de bienes y servicios a } \\
\text { las instituciones del Estado, así } \\
\text { como el seguimiento, monitoreo y } \\
\text { evaluación a los procedimientos y } \\
\text { procesos de selección y } \\
\text { contratación de proveedores. Se } \\
\text { encarga también de unificar los } \\
\text { requerimientos de bienes y } \\
\text { servicios a través del portal del } \\
\text { SEACE }\end{array}$ \\
\hline $\begin{array}{l}\text { Presupuesto } \\
\text { Público }\end{array}$ & $\begin{array}{l}\text { Ministerio de Economía y } \\
\text { Finanzas / Dirección } \\
\text { General del Presupuesto } \\
\text { Público }\end{array}$ & $\begin{array}{l}\text { Se enfoca en el proceso } \\
\text { presupuestario de las entidades } \\
\text { del sector estatal, brindando } \\
\text { asistencia técnica, seguimiento y } \\
\text { evaluación a las cinco fases en } \\
\text { las que se ha divido el proceso: } \\
\text { programación, formulación, } \\
\text { aprobación, ejecución } \\
\text { evaluación }\end{array}$ \\
\hline Tesorer & $\begin{array}{l}\text { Ministerio de Economía y } \\
\text { Finanzas / Dirección }\end{array}$ & $\begin{array}{l}\text { Centra su accionar en la } \\
\text { administración de los fondos }\end{array}$ \\
\hline
\end{tabular}




\begin{tabular}{|c|c|c|}
\hline & $\begin{array}{l}\text { General de } \\
\text { Endeudamiento y Tesoro } \\
\text { Público }\end{array}$ & $\begin{array}{l}\text { públicos en las entidades } \\
\text { estatales, para un uso eficaz y } \\
\text { eficiente de los recursos, sin } \\
\text { distinguir la fuente de } \\
\text { financiamiento de donde estos } \\
\text { fondos provengan }\end{array}$ \\
\hline $\begin{array}{l}\text { Endeudamiento } \\
\text { Público }\end{array}$ & $\begin{array}{l}\text { Ministerio de Economía y } \\
\text { Finanzas / Dirección } \\
\text { General de } \\
\text { Endeudamiento y Tesoro } \\
\text { Público }\end{array}$ & $\begin{array}{l}\text { Se encarga de regular los } \\
\text { procedimientos para un correcto } \\
\text { endeudamiento por parte de las } \\
\text { entidades estatales, ya sea con } \\
\text { entidades financieras nacionales } \\
\text { como internacionales }\end{array}$ \\
\hline $\begin{array}{l}\text { Contabilidad } \\
\text { Pública }\end{array}$ & $\begin{array}{l}\text { Ministerio de Economía y } \\
\text { Finanzas / Dirección } \\
\text { General de Contabilidad } \\
\text { Pública }\end{array}$ & $\begin{array}{l}\text { Lidera el sistema contable de las } \\
\text { entidades públicas, emitiendo } \\
\text { para ello normas y } \\
\text { procedimientos. Se entiende por } \\
\text { sistema contable a los ingresos, } \\
\text { egresos, pago de obligaciones y } \\
\text { otras actividades que se } \\
\text { relacionen con utilización y } \\
\text { administración de los fondos del } \\
\text { Estado }\end{array}$ \\
\hline $\begin{array}{l}\text { Inversión } \\
\text { Pública }\end{array}$ & $\begin{array}{l}\text { Ministerio de Economía y } \\
\text { Finanzas / Dirección } \\
\text { General de } \\
\text { Programación Multianual } \\
\text { del Sector Pública }\end{array}$ & $\begin{array}{l}\text { Regula las actividades } \\
\text { destinadas a la inversión estatal, } \\
\text { con la finalidad de optimizar el } \\
\text { uso de los recursos públicos. } \\
\text { Para un mejor desempeño y } \\
\text { control se ha creado Invierte.pe, } \\
\text { que establece las normas y } \\
\text { procedimientos aplicables en las } \\
\text { fases de pre inversión, inversión y } \\
\text { post inversión a las que están } \\
\text { sometidos los proyectos de } \\
\text { inversión pública }\end{array}$ \\
\hline $\begin{array}{l}\text { Planeamiento } \\
\text { Estratégico }\end{array}$ & $\begin{array}{l}\text { Presidencia del Consejo } \\
\text { de Ministros y } \\
\text { Nacional }\end{array}$ & $\begin{array}{l}\text { Regula la planificación de las } \\
\text { entidades públicas, para ello } \\
\text { emite las normas y }\end{array}$ \\
\hline
\end{tabular}




\begin{tabular}{|c|c|c|}
\hline & $\begin{array}{l}\text { Planeamiento } \\
\text { Estratégico }\end{array}$ & $\begin{array}{l}\text { procedimientos necesarios con la } \\
\text { finalidad de propiciar la } \\
\text { elaboración y posterior ejecución } \\
\text { de los planes de desarrollo, los } \\
\text { cuáles deben estar alineados } \\
\text { entre los tres niveles de gobierno }\end{array}$ \\
\hline $\begin{array}{l}\text { Defensa Judicial } \\
\text { del Estado }\end{array}$ & $\begin{array}{l}\text { Ministerio de Justicia y } \\
\text { Derechos Humanos y } \\
\text { Consejo de Defensa } \\
\text { Jurídica del Estado }\end{array}$ & $\begin{array}{l}\text { Es el responsable de la defensa } \\
\text { jurídica del Estado, mediante el } \\
\text { accionar de los Procuradores } \\
\text { Públicos. La defensa del Estado } \\
\text { se extiende desde los niveles sub } \\
\text { nacionales hasta los niveles } \\
\text { internacionales, evitando así } \\
\text { vulneraciones al país en los } \\
\text { fueros militar, judicial, arbitral, } \\
\text { constitucional, comercial, entre } \\
\text { otros. }\end{array}$ \\
\hline $\begin{array}{l}\text { Control } \\
\text { Gubernamental }\end{array}$ & $\begin{array}{l}\text { Contraloría General de la } \\
\text { República }\end{array}$ & $\begin{array}{l}\text { Está destinado a conducir el } \\
\text { ejercicio del control } \\
\text { gubernamental en las entidades } \\
\text { públicas y de manera } \\
\text { descentralizada. El objetivo es } \\
\text { promover y posibilitar el uso } \\
\text { racional y correcto de los } \\
\text { recursos públicos de que } \\
\text { disponen de las entidades del } \\
\text { Estado }\end{array}$ \\
\hline $\begin{array}{l}\text { Modernización } \\
\text { de la Gestión } \\
\text { Pública }\end{array}$ & $\begin{array}{l}\text { Presidencia del Consejo } \\
\text { de Ministros - Secretaría } \\
\text { de Gestión Pública }\end{array}$ & $\begin{array}{l}\text { Tiene como meta promover un } \\
\text { gobierno eficiente, abierto y } \\
\text { moderno, ubicado en el centro de } \\
\text { la población y como potenciador } \\
\text { de su desarrollo. Su accionar es } \\
\text { transversal a todas las entidades } \\
\text { del Estado, promoviendo el uso } \\
\text { intensivo de las tecnologías de la } \\
\text { información y el conocimiento }\end{array}$ \\
\hline
\end{tabular}

Fuente: Adaptado de Martínez (2015). 
El Congreso del Perú emitió la Ley N³0204, publicada el 11 de setiembre del 2018, que regula la transferencia de la gestión administrativa de los gobiernos regionales y gobiernos locales, como una norma que posibilita la transferencia de gestión administrativa con la finalidad de posibilitar la continuidad de la provisión de servicios a la comunidad, durante las transiciones de nuevas gestiones administrativas. Según la citada norma, las acciones de transferencia de gestión administrativa están a cargo de los gobernadores regionales y los alcaldes provinciales y distritales que cesan en sus cargos, quienes cederán la posta a las nuevas autoridades electas, proceso que debe realizase siguiendo los procedimientos y plazos establecidos por norma.

En el proceso de transferencia de gestión administrativa participan los siguientes actores: Las autoridades salientes y entrantes (gobernadores regionales y alcaldes provinciales y distritales), los funcionarios y servidores públicos que participan en el proceso de transferencia de gestión, los miembros del grupo de trabajo designados por la autoridad saliente, el equipo revisor designado por la autoridad electa y la Contraloría General de la República. La operatividad de la transferencia de gestión administrativa fue normada por la Contraloría General de la República, mediante la Directiva № 082018-CG/GTN, aprobada por Resolución de Contraloría N³48-2018-CG el año 2018, la cual se puede dividir en dos tipos: una primera por término regular del periodo de gestión y una segunda por consulta popular de revocatoria.

\section{MATERIALES Y MÉTODOS}

El método de la investigación que se presenta es del enfoque cualitativo con diseño inductivo, debido a que sobre la opinión de expertos se propondrá una tendencia o patrón conductual, y con el tipo de investigación básica, pues se orienta a la profundización y clarificación de la información conceptual sobre la administración pública. (Hernández et al., 2014). 
Para realizar la investigación se inició revisando la bibliografía existente sobre el tema de las transferencias de gestión administrativa de los gobiernos sub nacionales, luego se procedió a realizar un método Delphi en la ciudad de Chachapoyas con 20 servidores públicos (Casa y Folleco, 2019; García y Suárez, 2013; Ortega, 2008; Reguant-Álvarez y Torrado-Fonseca, 2016). El método Delphi se aplicó entre los meses de junio y julio del año 2020, con reuniones mediante la plataforma Zoom, la consolidación de las conclusiones finales se realizó en agosto del mismo año. Con la información obtenida en el Delphi, se procedió, a verificar los resultados y escuchar las recomendaciones de los especialistas en el tema. Finalmente, se recogieron todos estos resultados y se elaboró el borrador final, para luego compartirlo con expertos y elaborar los resultados finales de la investigación.

A continuación, se utilizó el método de Proceso de Análisis Jerárquico (AHP, del inglés Analytical Hierarchy Process) para evaluar cada una de las alternativas, utilizando para ello criterios de evaluación obtenidos a partir de factores sociales, económicos, institucionales y legales y luego definir una fecha de cambio de gestión que sea más beneficiosa para el país (Espinosa y Tigre, 2017; Saaty, 1984; Toskano, 2005).

Para determinar el tamaño muestral, se tuvo en cuenta es que los involucrados (servidores y expertos) hayan participado por lo menos una vez en procesos de transferencia administrativa de gobiernos regionales y/o locales en la región Amazonas.

Para la muestra se contó con la información que se recabó de veinte funcionarios con experiencia en cambios de gestión, y que hayan tenido cuando menos 6 meses de trabajo en las instituciones donde participaron en el cambio de gestión, ellos prestan y/o prestaron servicios en el gobierno regional de Amazonas, las municipalidades provinciales de Rodríguez de Mendoza y Chachapoyas, así como de las municipalidades distritales de Mariscal Benavides, Huancas y Olleros. La evaluación de la información se realizó con técnicas estadísticas no paramétricas. Debido al carácter de la investigación, se consideró como información sustentatoria las opiniones y 
resultados del Delphi realizado en la ciudad de Chachapoyas, por lo que los resultados, son la integración de las ideas con mayor aceptación y concertación por parte de los participantes. Para los cálculos se utilizó el paquete informático Microsoft Excel 2016.

\section{RESULTADOS}

\subsection{Resultados de la aplicación del Método Delphi}

Luego de evaluar los aportes de cada especialista invitado, se llegaron a los siguientes resultados:

\section{Sistema de Gestión de recursos humanos}

\section{Cambio de gestión administrativa el 31 de diciembre:}

Debilidades: - La gestión saliente podría contratar o renovar a todo el personal CAS por un año, por lo que se tendría que contar con personal no alineado a la gestión y entorpezca la atención a los ciudadanos. Para el caso de los servidores vinculados mediante el régimen del Decreto Legislativo №276, no habría riesgos observables. Para el caso de los colaboradores contratados como terceros (locación de servicios) tampoco habría problema, porque esta modalidad permite la contratación y culminación del contrato en cualquier momento del año.

- Normalmente, las gestiones entrantes buscan colocar profesionales de su entorno, podría suceder que los funcionarios que ya conocen y tienen experiencia en la entidad podrían ser separados sin capacitar y aleccionar al personal ingresante.

Fortalezas: - Las acciones adoptadas en materia de recursos humanos por las instituciones públicas se encuentran enmarcadas en la normativa vigente, por lo que se cumple con lo establecido en el marco legal. - La estabilidad de los trabajadores nombrados y contratados en el amparo del Decreto Legislativo $\mathrm{N}^{\circ} 276$, no genera dificultades para ninguna de las gestiones, entrante o saliente.

\section{Cambio de gestión administrativa el 28 de julio:}

Debilidades: - El personal CAS cuenta con contrato a tiempo variable, pero la gestión saliente podría usar las prerrogativas legales y contratar a las personas por todo el ejercicio presupuestal, dificultando el trabajo de las nuevas autoridades. Si a esta 
situación se suma que no exista presupuesto para contratar nuevo personal o que la entidad cuente con pocas plazas para personal de confianza, entonces la gestión entrante podría empezar con personal que aporte poco o que no participe en la política que proponga la gestión entrante.

Fortalezas: - Se consigue que la experiencia de los trabajadores sea utilizada en la institución, si se desea cambiar de personal, se tiene tiempo para capacitar a los ingresantes, sin perjudicar el normal desempeño de la entidad. - Aun cuando se trabaje con personal que provenga de la gestión anterior, es posible evaluar el desempeño de los trabajadores y determinar su continuidad en los demás años fiscales. Esto significa trabajar con los mejores técnicos, sin importar su procedencia política y en beneficio de la entidad y la población para la que se esté sirviendo.

\section{Sistema de Abastecimiento}

\section{Cambio de gestión administrativa el 31 de diciembre:}

Debilidades: - En muchos gobiernos locales y regionales, al conocerse los resultados de las elecciones y al no ganar un sucesor del partido o con algún tipo de vinculación con la gestión saliente, se abandona la prestación de los servicios básicos, los cuales generan caos y en algunos casos riesgos de contaminación. - Según la normatividad vigente, las fases del gasto público son: certificación, compromiso, devengado, girado y pagado. Para el devengado ya se debe tener el bien o servicio y la correspondiente conformidad, con lo cual la deuda queda oficialmente reconocida y el pago es mero trámite. Sin embargo, algunas gestiones entrantes aun cuando encuentran expedientes para pago debidamente devengados, se reúsan a girar el pago, incumpliendo el reconocimiento de las obligaciones con los proveedores, los cuales tienen que seguir un proceso para el reconocimiento de la deuda, que en algunos casos superan los seis meses de trámite. - En otro extremo, debido a que se tiene que devengar los expedientes para pago hasta el 31 de diciembre, algunas gestiones realizan esta actividad sin contar con la documentación sustentatoria pertinente e incluso sin que los bienes sean 
entregados o los servicios se hayan realizado, esto podría generar, por un lado, que se realicen pagos por bienes y servicios no entregados, o que la nueva gestión decida investigar y no realizar estos pagos, con lo que se podrían generar acciones legales que involucren a funcionarios y proveedores.

Fortalezas: - Exigencia para que la programación de gastos se realice dentro del año fiscal y se controle su ejecución, para así evitar la reversión de los recursos económicos que son exiguos en los gobiernos sub nacionales. - Condiciones necesarias para que las gestiones se acomoden a realizar todo el gasto en un año fiscal, pudiendo cumplir con su programación presupuestal.

\section{Cambio de gestión administrativa el 28 de julio:}

Debilidades: - Incumplimiento de plazos en los procesos de selección de proveedores, los cuáles podrían provocar reprogramaciones o que generen demandas legales a la entidad convocante.

Fortalezas: - Seguimiento efectivo a la contratación y recibimiento de los bienes y servicios requeridos por la institución pública, buscando la continuidad del aprovisionamiento en todo el año. - Reprogramación para cumplir con el Plan Anual de Adquisiciones y Contrataciones, re direccionar el rumbo y evaluar los procesos de selección realizados por la gestión anterior. - Riesgo mínimo para no reconocer deudas ficticias de la gestión anterior y posibilidad de verificar la entrega de bienes y servicios, esto significa el reconocimiento efectivo de las deudas generadas y el pago por bienes y servicios efectivamente entregados.

\section{Sistema de Presupuesto público}

\section{Cambio de gestión administrativa el 31 de diciembre:}

Debilidades: - Trabajar con el presupuesto programado por la gestión anterior, la cual tuvo una visión diferente a la actual.

Fortalezas: - Condiciones necesarias para el cumplimiento de la normativa vigente. 


\section{Cambio de gestión administrativa el 28 de julio:}

Debilidades: - Poco tiempo para reprogramar el presupuesto, lo cual significaría no ejecutar al máximo posible por la entidad.

Fortalezas: - Posibilidad de programar el presupuesto del año fiscal siguiente alineado a las políticas públicas que proponga la gestión entrante. - Posibilidad de alinear el presupuesto con la nueva gestión del gobierno regional, principal socio estratégico de los gobiernos sub nacionales.

\section{Sistema de Tesorería}

\section{Cambio de gestión administrativa el 31 de diciembre:}

Debilidades: - Probabilidad de no cumplir con el giro dentro del plazo establecido, debido a que los expedientes de pago (provenientes de Órdenes de Compra, Órdenes de Servicio y Contratos) se encuentren incompletos o con conformidades pendientes. No disponibilidad de Calendario en el mes de diciembre para la ejecución de los giros correspondientes.

Fortalezas: - Sistema contable programado para evaluar el gasto en cada Año Fiscal.

\section{Cambio de gestión administrativa el 28 de julio:}

Debilidades: - Desconocimiento de las actividades realizadas en la gestión saliente, por lo que los pagos podrían demorar más de lo necesario. - Expediente para pago incompletos, que podría dificultar el pago a proveedores.

Fortalezas: - Posibilidad de efectuar fiscalizaciones de oficio a los pagos realizados en la primera mitad del año. - Posibilidad de reprogramar los pagos del segundo semestre sin perjudicar a los proveedores y colaboradores de la entidad.

\section{Sistema de Contabilidad Pública}

Cambio de gestión administrativa el 31 de diciembre: 
Debilidades: - Probabilidad de gastos sin todas las fases completas: certificación, compromiso anual, compromiso mensual, devengado, giro y pago.

Fortalezas: - Sistema contable programado para evaluar el gasto en cada Año Fiscal.

\section{Cambio de gestión administrativa el 28 de julio:}

Debilidades: - Probabilidad de encontrar cierres contables con deficiencias o sin soporte documentario.

Fortalezas: - Probabilidad de auditar la contabilidad de la institución a tiempo, para tomar decisiones acertadas y evitar multas o sanciones tanto por parte del Gobierno Central como de acreedores y proveedores.

\section{Sistema de Inversión pública}

\section{Cambio de gestión administrativa el 31 de diciembre:}

Debilidades: - Limitación en la continuidad de obras públicas. Algunas veces se demora el pago de los contratistas. - Obras inconclusas, con gastos importantes de dinero, que se encuentran en estado de abandono. - Deficiencia en la liquidación de las obras, debido a que la gestión saliente no entrega toda la documentación completa del PIP u obra en ejecución.

Fortalezas: - Posibilidad de ejecutar todo lo programado en el Año Fiscal anterior, es decir contar con un punto de partida.

\section{Cambio de gestión administrativa el 28 de julio:}

Debilidades: - Probabilidad de retraso en el pago de valorizaciones, debido a que la nueva gestión podría averiguar los antecedentes antes de regularizar los pagos.

Fortalezas: - Probabilidad de redefinir objetivos y analizar la calidad del gasto público manifestado en proyectos de inversión pública. - Evaluar el cumplimiento de los contratos, así como el avance físico y financiero de las obras. - Tiempo suficiente para 
evaluar el presupuesto del siguiente año, y organizarlo de acuerdo a las nuevas exigencias y condiciones económicas, sociales, ambientales, etc.

\section{Sistema de Planeamiento Estratégico}

\section{Cambio de gestión administrativa el 31 de diciembre:}

Debilidades: - Con frecuencia, algunos gobiernos planifican pensando solo el último año de gestión, no poniendo énfasis en el siguiente año, por lo que se priorizan obras que no son necesarias o se recargan de obras que no impactarán en la población. Determinación del presupuesto sobre una realidad propia, desconociendo los objetivos y metas de la siguiente gestión, que iniciará funciones en el siguiente año.

Fortalezas: - Alineación con la ejecución del gasto público, que utiliza años fiscales (01 de enero al 31 de diciembre) para su programación y evaluación. - Posibilidad de no empezar de cero y utilizar la programación que dejó la gestión anterior.

\section{Cambio de gestión administrativa el 28 de julio:}

Debilidades: - Posibilidad de programar erróneamente el presupuesto para el siguiente año, debido a que el inicio de la gestión podría coincidir con el cierre de la programación del presupuesto público del siguiente año.

Fortalezas: - Posibilidad de evaluar la ejecución del presupuesto del año fiscal en curso, redefinirla y distribuirla para ejecutar la mayor cantidad posible con la mejor calidad de gasto posible. - Posibilidad de alinear el presupuesto con el Plan de Gobierno de la gestión entrante, pudiendo de esta manera cumplir con las metas y objetivos establecidos antes de ingresar a la gestión.

\section{Los Sistemas de Endeudamiento Público, Defensa judicial del Estado, Control}

Gubernamental, Sistema de Modernización de la gestión pública:

No se evidencian impactos significativos en estos Sistemas Administrativos. 


\subsection{Resultados de la aplicación del Método de Proceso de Análisis Jerárquico}

Para evaluar la propuesta se procedió a aplicar el método AHP. Se utilizó como insumos las fortalezas y debilidades evidenciadas por el Análisis Delphi en cada uno de los Sistemas Administrativos que evidencian un impacto en el cambio de fecha del cambio de gestión en los gobiernos locales y regionales.

La identificación del problema consistió en desear conocer, sobre el análisis realizado con los expertos, cuál de las alternativas generará mayor bienestar en la población y optimizará el uso de los recursos públicos. El objetivo que se perseguía era seleccionar la mejor fecha para la realización del cambio de gestión de gobiernos locales y regionales peruanos. Para ello se identificaron los siguientes criterios:

a. Factores sociales

a.1. Continuidad en la provisión de bienes y servicios a la población: Proporcionar servicios adecuados y sin interrupción.

a.2. Percepción de la población hacia sus autoridades: Crear canales de comunicación eficientes y dar a conocer la gestión institucional.

b. Factores económicos

b.1. Ejecución del presupuesto institucional: Capacidad de manejo del presupuesto y el manejo responsable de las deudas y obligaciones.

b.2. Calidad del gasto: Adquirir bienes y servicios necesarios, con eficiencia y eficacia.

b.3. Captación de ingresos monetarios: Capacidad para potenciar y optimizar la captación de recursos directamente recaudados y el cobro de impuestos.

c. Factores institucionales

c.1. Articulación interinstitucional: Capacidad para utilizar adecuadamente las fortalezas y oportunidades institucionales, en coordinación con otras instituciones locales. 
c.2. Conocimiento específico para el desempeño institucional: Capacidad para contar con persona adecuadamente calificado, que sepa que hacer.

d. Factores legales

d.1. Articulación con la normatividad vigente: Capacidad para informar las normativas a la población y cumplir con las metas y directrices vigentes.

d.2. Cumplimiento de obligaciones contraídas: Pago de proveedores, continuidad de obras públicas.

También se Identificaron las alternativas que serían comparadas:

Alternativa 1: Realizar el cambio de gestión de gobiernos locales y regionales peruanos el 31 de diciembre.

Alternativa 2: Realizar el cambio de gestión de gobiernos locales y regionales peruanos el 28 de julio.

Se inició construyendo la matriz de comparación por pares de los criterios (Tabla 2). La cual resume la preferencia entre criterios. La diagonal es 1 porque al comparar un criterio consigo mismo, siempre será igual de preferida. Al comparar dos criterios distintos tendremos que, si es mayor que 1, ese criterio (de la primera columna) es preferido al otro criterio (de la primera fila). Por ejemplo, al comprar a1 y a2, tenemos que la preferencia es 6 , o sea que a1 es fuertemente preferida a a2, según la escala de comparación de Saaty ${ }^{1}$.

\section{Tabla 2}

Matriz de comparación por pares de los criterios

\begin{tabular}{cccccccccc}
\hline & $\mathrm{a} 1$ & $\mathrm{a} 2$ & $\mathrm{~b} 1$ & $\mathrm{~b} 2$ & $\mathrm{~b} 3$ & $\mathrm{c} 1$ & $\mathrm{c} 2$ & $\mathrm{~d} 1$ & $\mathrm{~d} 2$ \\
\hline $\mathrm{a} 1$ & 1 & 6 & 2 & 1 & 4 & 6 & 3 & 2 & 2 \\
$\mathrm{a} 2$ & $1 / 6$ & 1 & $1 / 4$ & $1 / 6$ & $1 / 3$ & $1 / 2$ & $1 / 3$ & $1 / 5$ & $1 / 4$ \\
$\mathrm{~b} 1$ & $1 / 2$ & 4 & 1 & $1 / 2$ & 3 & 5 & 2 & 1 & 2
\end{tabular}

\footnotetext{
${ }^{1}$ Saaty (1984) propuso una escala de comparación que va de 1 a 9, con 1 = ambos criterios son igualmente preferidos, 3 = El primer criterio moderadamente preferido, 5 = el primer criterio fuertemente preferido, $7=$ el primer criterio muy fuertemente preferido, $9=$ el primer criterio extremadamente preferido. Los valores $2,4,6,8$ son valores intermedios de preferencia.
} 


\begin{tabular}{cccccccccc}
$\mathrm{b} 2$ & 1 & 6 & 2 & 1 & 4 & 6 & 3 & 2 & 2 \\
$\mathrm{~b} 3$ & $1 / 4$ & 3 & $1 / 3$ & $1 / 4$ & 1 & 3 & $1 / 2$ & $1 / 4$ & $1 / 3$ \\
$\mathrm{c} 1$ & $1 / 6$ & 2 & $1 / 5$ & $1 / 6$ & $1 / 3$ & 1 & $1 / 4$ & $1 / 6$ & $1 / 5$ \\
$\mathrm{c} 2$ & $1 / 3$ & 3 & $1 / 2$ & $1 / 3$ & 2 & 4 & 1 & $1 / 5$ & $1 / 4$ \\
$\mathrm{~d} 1$ & $1 / 2$ & 5 & 1 & $1 / 2$ & 4 & 6 & 5 & 1 & 2 \\
$\mathrm{~d} 2$ & $1 / 2$ & 4 & $1 / 2$ & $1 / 2$ & 3 & 5 & 4 & $1 / 2$ & 1 \\
\hline
\end{tabular}

Fuente: elaborado por el autor.

A continuación, se normalizó la tabla, es decir se dividió cada elemento entre la suma total de la columna a la cual pertenece. Obtenidos todos los valores normalizados se suma todas las cantidades de la misma fila, obteniéndose el peso relativo de cada criterio, que se presenta en la Tabla 3. En la referida tabla se puede observar que los criterios más importantes son: a1 y b2 con 0.20 de peso cada uno. El tercer criterio más importante es d1 con 0.17 de peso. Estos tres criterios definen el $57 \%$ de la decisión al momento de evaluar las alternativas.

\section{Tabla 3}

Matriz con el peso relativo de los criterios

\begin{tabular}{clc}
\hline$N^{\circ}$ & \multicolumn{1}{c}{ Criterio } & Peso \\
\hline a1 & Continuidad en la provisión de bienes y servicios a la población & $\mathbf{0 . 2 0}$ \\
a2 & Percepción de la población hacia sus autoridades & $\mathbf{0 . 0 3}$ \\
b1 & Ejecución del presupuesto institucional & $\mathbf{0 . 1 3}$ \\
b2 & Calidad del gasto & $\mathbf{0 . 2 0}$ \\
b3 & Captación de ingresos monetarios & $\mathbf{0 . 0 5}$ \\
c1 & Articulación interinstitucional & $\mathbf{0 . 0 3}$ \\
c2 & Conocimiento específico para el desempeño institucional & $\mathbf{0 . 0 7}$ \\
d1 & Articulación con la normatividad vigente & $\mathbf{0 . 1 7}$ \\
d2 & Cumplimiento de obligaciones contraídas & $\mathbf{0 . 1 2}$
\end{tabular}

Fuente: elaborado por el autor.

Ahora que hemos establecido la prioridad de los criterios, podemos jerarquizar la prioridad de las alternativas, es decir, trasladaremos la prioridad de cada alternativa respecto a cada criterio mostrándolo numéricamente. Para priorizar las alternativas, volvemos a la comparación pareada con la escala numérica de Saaty. Esta vez compararemos de dos en dos las distintas alternativas respecto a cada criterio. 
Con los cálculos previos realizados, se buscó determinar que alternativa convendría materializar. Para realizar esta fase de la aplicación del método AHP, se recogieron los promedios de las filas normalizadas de todas las matrices que evalúan la importancia relativa, respecto a cada criterio, presentando los resultados en la Tabla 4.

\section{Tabla 4}

Consolidado de la evaluación de alternativas

\begin{tabular}{cccccccccc}
\hline & $\mathrm{a} 1$ & $\mathrm{a} 2$ & $\mathrm{~b} 1$ & $\mathrm{~b} 2$ & $\mathrm{~b} 3$ & $\mathrm{c} 1$ & $\mathrm{c} 2$ & $\mathrm{~d} 1$ & $\mathrm{~d} 2$ \\
\hline Alternativa 1 & 0.75 & 0.63 & 0.42 & 0.88 & 0.63 & 0.75 & 0.42 & 0.41 & 0.75 \\
Alternativa 2 & 0.25 & 0.31 & 1.25 & 0.22 & 0.31 & 0.25 & 1.25 & 1.63 & 0.25 \\
\hline
\end{tabular}

Fuente: elaborado por el autor.

Luego, se procedió a multiplicar esta matriz con la matriz de los pesos relativos de los criterios, establecidos en la Tabla 3. Se obtuvo el resultado que se muestra en la Tabla 5, con la cual se puede concluir que, es preferible elegir la alternativa 2, y realizar el cambio de gestión de gobiernos locales y regionales peruanos el 28 de julio, favoreciendo así de mejor manera a la población.

\section{Tabla 5}

Vector de prioridad de las alternativas

\begin{tabular}{lc}
\hline \multicolumn{1}{c}{ Alternativas } & Prioridad \\
\hline Alternativa 1: Realizar el cambio de gestión de gobiernos locales y & $\mathbf{0 . 6 4 5}$ \\
regionales peruanos el 31 de diciembre & \\
Alternativa 2: Realizar el cambio de gestión de gobiernos locales y & $\mathbf{0 . 6 8 0}$ \\
regionales peruanos el 28 de julio & \\
\hline Fuente: elaborado por el autor. &
\end{tabular}

Es importante resaltar que los resultados obtenidos se deben enteramente a los criterios establecidos, si se eligen otros criterios, se pueden generar resultados totalmente diferentes. 


\section{DISCUSIÓN}

En el Perú se ha conseguido importantes avances en materia de descentralización política, sin embargo, esta no ha ido acompañada de cambios en materia tributaria y de transferencias de recursos, tampoco de una estrategia coherente para aumentar las habilidades y capacidades a nivel sub nacional, tal como lo afirman otros estudios similares (Abanto, 2017; López, 2019; Rojas, 2015).

Los sistemas administrativos del Estado peruano son los conjuntos de principios, normas, procedimientos, técnicas e instrumentos que sirven de base para que las actividades de la administración pública se organicen. Para su realización se requiere que varias entidades los ejecuten, siguiendo los lineamientos emitidos por el Ente rector, definido al momento de establecerse el sistema, como también lo define Acosta (2013). En el caso particular del Sistema de Abastecimiento, Enciso et al (2018) concluyen en el estudio del cumplimiento de este sistema para la región Ayacucho que, en la evaluación del cumplimiento de la prestación de servicios, el 73,6\% de los jefes de oficinas de Abastecimiento entrevistados considera que fue regular y solo el $11,1 \%$ de ellos considera que fue adecuada. Considerando que el estudio fue realizado en el último año de gestión administrativa, se puede predecir una herencia complicada para la nueva gestión, tal como se afirma en el presente estudio.

Se ha evidenciado empíricamente y con la aplicación de dos métodos de análisis, que es recomendable realizar el cambio de gestión administrativa de los gobiernos locales y regionales peruanos el 28 de julio, después de cuatro años de gestión de la gestión saliente. Con esto, coincidimos con Leonarte (2018) al mencionar que la transferencia de gestión administrativa debe permitir la continuidad de las labores de los gobiernos regionales y locales, sin interrumpir el servicio al ciudadano.

La transferencia de gestión no debería normarse solamente al final de la gestión, sino debería promover un buen inicio de la gestión entrante, como lo recomienda 
también Leonarte (2018). Esta acción implicaría tomar conocimiento de las principales actividades a desarrollar, así como generar una agenda inicial para la nueva gestión.

\section{CONCLUSIONES}

Un adecuado cambio de gestión de los gobiernos locales y provinciales en la región Amazonas incidirá notablemente en el desempeño de los sistemas administrativos de la institución, a través de la provisión de servicios que ofrecen a la ciudadanía. Es posible modelar una manera en la cual el cambio de gestión administrativa posibilitará minimizar el impacto negativo de las transiciones de la gestión entrante, como caso particular se utilizó el método Delphi y el método de Proceso de Análisis Jerárquico (AHP).

A partir de la experiencia en la región Amazonas, en la investigación se demostró que con un cambio de gestión administrativa para el 28 de julio, se logra que las gestiones entrantes puedan tener mayor capacidad de administrar los sistemas administrativos en las instituciones que lideran. El cambio de gestión administrativa afecta notablemente a siete sistemas administrativos (Gestión de Recursos Humanos, Abastecimiento, Presupuesto Público, Tesorería, Contabilidad, Inversión Pública y Planeamiento Estratégico) y en cuatro de ellos, no tendrían un impacto significativo.

\section{REFERENCIAS BIBLIOGRÁFICAS}

Abanto, C. D. (2017). Descentralización fiscal y gestión local en el Perú. The Latin American and Iberian Law and Economics Association. Universiad del Pacífico. $\underline{\text { https://bit.ly/3fMvzuf }}$

Acosta, C. (2013). Ámbito de desarrollo de los sistemas administrativos. Actualidad Gubernamental, 52, II1-II4. Instituto Pacífico S.A.C.

Arroyo, J. (2017). ¿Ha tenido la descentralización un efecto importante en el desarrollo de las regiones? El caso peruano 2002-2015. Revista del CLAD Reforma y Democracia, 69 (2017), 159-190. https://bit.ly/3vklFW1 
Asela, J. (2015). La descentralización en Colombia, ¿realidad o espejismo? Consideraciones sobre la actualidad del proceso. Revista CEA, 1(1), 65-74. DOI: https://doi.org/10.22430/24223182.63

Casa, C. A. y Folleco, C. M. (2019). El método Delphi, prospectiva en ciencias sociales a través de varios casos prácticos: una revisión bibliográfica de los últimos 10 años. Revista Digital Tambara, 7 (37), 500-515. https://bit.ly/3bZ6s6m

Claros, R. (24 de octubre del 2014). El proceso de transferencia de gestión en los gobiernos subnacionales [Mensaje en un blog]. https://bit.ly/34j8t9h

Congreso Constituyente Democrático. (1993). Constitución Política del Perú. Congreso de la República del Perú.

Congreso del Perú (1997). Ley N²26864, Ley de Elecciones Municipales. Diario Oficial El Peruano. Lima: Congreso de la República del Perú, 13 de octubre.

Congreso del Perú (1998). Ley $N^{\circ}$ 26997, Ley que establece la conformación de comisiones de transferencia de la administración municipal. Diario Oficial El Peruano. Lima: Congreso de la República del Perú, 25 de noviembre.

Congreso del Perú (2002). Ley N²7683, Ley de Elecciones Regionales. Diario Oficial El Peruano. Lima: Congreso de la República del Perú, 14 de marzo.

Congreso del Perú (2002). Ley № 27867, Ley Orgánica de Gobiernos Regionales. Diario Oficial El Peruano. Lima: Congreso de la República del Perú, 16 de noviembre.

Congreso del Perú (2003). Ley N²7972, Ley Orgánica de Municipalidades. Diario Oficial El Peruano. Lima: Congreso de la República del Perú, 26 de mayo.

Congreso del Perú (2014). Ley N³0204, Ley que regula la transferencia de la gestión administrativa de gobiernos regionales y gobiernos locales, y establece medidas para contribuir a garantizar la continuidad de la provisión de servicios públicos, durante el proceso de transferencia de la gestión administrativa de gobiernos regionales y gobiernos locales. Diario Oficial El Peruano. Lima: Congreso de la República del Perú, 11 de junio. 
Congreso del Perú (2018). Decreto Legislativo № 1440, Del Sistema Nacional de Presupuesto Público. Diario Oficial El Peruano. Lima: Congreso de la República del Perú, 16 de setiembre.

Contraloría General de la República. (2018). Resolución de Contraloría № 348-2018CG. http://www.contraloria.gob.pe

De La Paz, J. G. (2017). Introducción a la Administración Pública Mexicana. Instituto Nacional de Transparencia, Acceso a la Información y Protección de Datos Personales (INAI). https://bit.ly/2SzLkfO

Definición.de (01 de marzo del 2021). Definición de Administración. https://definicion.de/administracion/

Enciso, E. R., Garay, K. Q., Gómez, J. y Martínez, M. (2018). Cumplimiento del sistema de abastecimientos, y sus efectos en las entidades del estado en Ayacucho. Revista Gobierno y Gestión Pública, 5 (2), 51-73. https://doi.org/10.24265/iggp. $\underline{2018 . v 5 n 2.04}$

Espinosa, M. y Tigre, F. (2017). Proceso Analítico Jerárquico (AHP) Aplicado a la

Selección de Metodología de Gestión: Caso CORFOPYM. http://192.188.46.193/ handle/123456789/47960

García, M. y Suárez, M. (2013). El método Delphi para la consulta a expertos en la investigación científica. Revista Cubana de Salud Pública, 39 (2), 253-267. https://bit.ly/3i0AcDx

Hernández, R., Fernández, C. y Baptista, M. D. (2014). Metodología de la investigación (6ta ed.). McGraw-Hill Interamericana. https://bit.ly/3yl5IMn

Leonarte, J. C. (2018). Transferencia de la gestión administrativa de gobiernos regionales y gobiernos locales: La importancia de la continuidad del servicio: Conocimiento y aplicación por parte de autoridades salientes y entrante, servidores y funcionarios públicos. Policy Brief $N^{\circ} 04$. Universidad del Pacífico. https://bit.ly/3vrK1hQ 
López, D. N. (2019). Propuesta de políticas de Gestión Municipal para la Reducción de la desnutrición infantil en la Municipalidad Distrital de Ocros, Ocros - 2018. Revista Gobierno y Gestión Pública, 6 (1), 143-159. https://doi.org/10.24265/IGGP. 2019.v6.n1.07

Martínez, A. (2015). Manual de los sistemas administrativo del sector público. Gaceta Jurídica. https://bit.ly/3yFdel0

Organisation for Economic Co-operation and Development. (2017). OECD Territorial Reviews: Peru 2016. OECD. DOI: https://dx.doi.org/10.1787/9789264262904-en

Ortega, F. (2008). El método Delphi, prospectiva en ciencias sociales a través del análisis de un caso práctico. Revista EAN, 64, 31-54. http://www.redalyc.org/articulo.oa?id=20612981004

R\&C Consulting. (01 de marzo del 2021). ¿Cuáles son los sistemas administrativos y entes rectores del Perú?. https://bit.ly/2SwJgVU

Reguant-Álvarez, M. y Torrado-Fonseca, M. (2016). El método Delphi. REIRE, Revista d'Innovació $\quad i \quad$ Recerca en Educació, $9 \quad$ (1), 87-102. https://doi.org/10.1344/reire2016.9.1916.

Rojas, P. (2015). Administración pública y los principios del derecho administrativo en Perú. Revista Digital de Derecho Administrativo, 13 (Enero-Junio), 193-209. https://bit.ly/3flY9fU

Saaty T.L. (1984) The Analytic Hierarchy Process: Decision Making in Complex Environments. In: R. Avenhaus \& R. K. Huber (Eds.) Quantitative Assessment in Arms Control (pp. 285-308). Boston, MA: Springer. https://doi.org/10.1007/978-1$\underline{4613-2805-6 \quad 12}$

Toskano, G. B. (2005). El Proceso de Análisis Jerárquico (AHP) como herramienta para la toma de decisions en la selección de proveedores [Tesis de Pregrado]. Universidad Nacional Mayor de San Marcos. https://bit.ly/34snNQF 